\title{
Diferentes Protocolos de Grabado Ácido en Dentina; Estudio Micromorfológico.
}

\section{Different protocols for acid etching in dentin; micromorphological study}

\begin{abstract}
Blas Galdames ${ }^{1 *}$, Mabel Brunoto², Natalia Marcus', Fernando Grandon', Elba Priotto²
1. DDS, PhD Departamento Odontología Restauradora, Universidad de Concepción, Chile. 2. $D D S, P h D$ Universidad Nacional de Córdoba, Argentina

${ }^{*}$ Correspondencia autor: Blas Galdames Dirección postal: Facultad de Odontología Universidad de Concepción, Roosevelt 1550 Concepción, Chile. | Teléfono +56 4122204481 | Email: galdamesb@gmail.com

Trabajo recibido el 29/06/2017. Aprobado para su publicación el 29/10/2017

\section{RESUMEN}

El objetivo fue evaluar las características de túbulos dentinarios expuestos por acción de diferentes protocolos de grabado en distintas zonas dentinarias. Se midió densidad tubular y diámetro. Se estudiaron 60 primeros premolares humanos sanos sometidos a tratamiento de endodoncia. Se realizaron cortes de las raíces a $5 \mathrm{~mm}$ del límite amelocementario y en sentido longitudinal bucolingual, obteniendo mitades iguales (mesial y distal) que se agruparon según tratamiento acondicionador a recibir: Control, EDTA, $\mathrm{H}_{3} \mathrm{PO}_{4} 15$ y 45 seg., y $\mathrm{H}_{3} \mathrm{PO}_{4} 15$ y 45 seg. + NaOCl. Se realizó análisis en MEB en tres sectores: dentina coronaria, cámara pulpar e inicio del conducto radicular. La comparación entre las secciones se analizó mediante prueba t de Student para datos apareados, fijando valor $p<0,05$ para significación estadística. Se utilizó programa Infostat versión profesional 2009. No se observaron diferencias significativas en los grupos al comparar densidad tubular para las tres zonas estudiadas. Al comparar diámetro de los túbulos, se obtuvieron valores mayores cuando se acondicionó con ácido ortofosfórico al $45 \%+\mathrm{NaOCl}$. Los resultados ponen de manifiesto el comportamiento clínico heterogéneo de las distintas zonas dentinarias luego de diferentes protocolos para adhesión con técnica de grabado total. Protocolo tradicional de acondicionamiento con ácido ortofosfórico al $35 \%$ por 15 segundos no asegura grabado uniforme de las tres zonas dentinarias estudiadas. Protocolos donde se aumenta el tiempo de grabado y/o se incorpora $\mathrm{NaOCl}$ al $5 \%$ por un minuto como agente desproteinizante podrían permitir patrones más predecibles y apropiados de grabado en las tres zonas estudiadas de dientes endodonciados.
\end{abstract}

PALABRAS CLAVE

Adhesión dentinaria; Grabado ácido; Permeabilidad dentinaria.

Rev. Clin. Periodoncia Implantol. Rehabil. Oral Vol. 11(2); 91-97, 2018.

\section{ABSTRACT}

Evaluate in endodontically treated teeth the effects of different etching protocols on dentinal tubule exposure and diameter at three dentin zones. Sixty healthy firstpremolars from human volunteers were studied. Each tooth received endodontictreatment. Teeth were transversely sectioned $5 \mathrm{~mm}$ below amelocemental junction andlongitudinaly sectioned in the buccolingual direction, obtaining two crown halves. Eachcrown half was grouped according to etching protocol into: Control, EDTA, $\mathrm{H}_{3} \mathrm{PO}_{4} 15$ and 45 sec., e $\mathrm{H}_{3} \mathrm{PO}_{4} 15$ and 45 seg. $+\mathrm{NaOCl}$. Three dentinal areas were analyzed withscanning electron microscope (SEM) within each tooth: coronal dentin, pulp chamber dentin and the beginning of the root canal. Comparison between dentin zones for each treatment was made with Student's t-test for paired data, using $\mathrm{P}<0.05$ for statisticalsignificance. No significant differences in dentinal tubule exposure within the threestudied zones were found. However, when comparing tubule diameter, higher valueswere obtained at the three dentin zones studied when $\mathrm{H}_{3} \mathrm{PO}_{4}$ and $\mathrm{NaOCl}$ wer usedin combination. The results of this study showed heterogeneous behavior of differentdentin zones after exposing them to various total etch protocols. The use of $37 \% \mathrm{H}_{3} \mathrm{PO}_{4}$ for $15 \mathrm{~s}$ did not ensure a uniform etch in the three studied zones. Protocols with $\mathrm{H}_{3} \mathrm{PO}_{4}$ for $45 \mathrm{~s}$ with $5 \% \mathrm{NaOCl}$ for $1 \mathrm{~min}$, incorporated as a deproteinizing agent, couldallow more predictable and appropriate etching patterns in endodontically treated teeth.

\section{KEYWORDS}

Dental bonding; acid etching; dentin permeability.

Rev. Clin. Periodoncia Implantol. Rehabil. Oral Vol. 11(2); 91-97, 2018. 


\section{INTRODUCCIÓN}

Para restaurar un elemento dentario utilizando sistemas adhesivos, además de lograr una buena adhesión entre biomateriales restauradores y esmalte dental, resulta primordial la adhesión de éstos con el sustrato dentinario. Este fenómeno permite un buen sellado marginal, reduciendo la microfiltración y alargando la longevidad de las restauraciones ${ }^{(1)}$

A partir de la técnica de grabado total introducida por Fusayama et al. ${ }^{(2)}$, se han propuesto distintas mejoras en los protocolos y sistemas adhesivos diseñados para este fin. Esto ha generado nuevas posibilidades para las restauraciones adhesivas, incluyendo resultados favorables en dientes tratados endodónticamente, con innumerables ventajas frente a materiales tradicionales no adhesivos ${ }^{(3)}$, debido a que una resina unida al diente permite la trasmisión del estrés funcional a través de la interfase de unión, con un potencial refuerzo a la estructura del diente ${ }^{(4)}$.

A pesar que los valores de adhesión logrados en dentina coronaria son bastante buenos $^{(5)}$, comparables en algunos casos a esmalte, cambios en áreas relativas de la superficie dentinaria pueden afectar la adhesión en forma relevante. La dificultad en la unión de un material adhesivo a la estructura dentinaria está asociada a su estructura histológicamente compleja $^{(6)}$. En esta línea, Causton ${ }^{(7)}$ y Giannini y col. ${ }^{(8)}$ encontraron diferencias en la fuerza de unión en dentina coronaria, siendo más baja en dentina profunda comparada con dentina superficial.

Tanumiharja y col. ${ }^{(9)}$, utilizando test de microtracción, también concordaron en la heterogeneidad de los valores de adhesión a dentina, demostrando diferencias significativas en la resistencia de unión a dentina oclusal versus dentina del piso pulpar.

Cagidiaco y col.(10) evaluaron la micromorfología de la dentina en términos de orientación y densidad de los túbulos dentinarios e incremento del área de superficie acondicionada, demostrando que el aumento de área de superficie dentinaria intertubular y peritubular es responsable del aumento de la fuerza de unión después del grabado dentinario. Los túbulos dentinarios tienen una dirección, densidad y diámetro que es conocido(11), y su dirección en las paredes de la cavidad depende de su localización ${ }^{(10)}$

Esto da a entender que la microestructura regional de la dentina puede tener importancia cuando se requiera lograr máximos valores de adhesión, especialmente cuando se trata de dientes endodonciados que se pretenden restaurar en forma conservadora y en donde se exponen zonas dentinarias diferentes como son: dentina coronal, dentina cameral y de la zona cervical del conducto radicular.

El objetivo de este trabajo es estudiar los cambios en densidad y diámetro de los túbulos dentinarios, en tres zonas de premolares humanos tratados endodónticamente, cuando son sometidos a diferentes protocolos de grabado.

\section{MATERIALES Y MÉTODOS}

Se utilizaron sesenta primeros premolares superiores humanos sanos, sin restauración, extraídos por fines ortodóncicos en pacientes entre 15 y 25 años, considerando como criterios de inclusión (revisado clínica y radiográficamente): a.- premolares bicanaliculares y b.bifurcación del conducto definida y que se inicia en el tercio coronal de la raíz. El procedimiento de recolección de los especímenes fue autorizado por el Comité de Ética de la Facultad de Odontología de la Universidad de Concepción y por los pacientes, mediante firma de Consentimiento Informado.

Todas las piezas se almacenaron sumergidas en solución de formol neutro al $10 \%$, para luego ser divididas en forma aleatoria en un grupo control y cinco grupos experimentales con diez especímenes cada uno.

\section{PREPARACIÓN DE LAS MUESTRAS}

\section{Grupos control:}

A diez dientes se practicó accesos endodónticos e instrumentación de los conductos siguiendo la técnica que se describe para los subgrupos experimentales, pero sin realizar la obturación endodóntica del canal radicular.

\section{Grupos experimentales:}

Se realizó la trepanación de cada diente de manera conservadora. E tratamiento radicular de todos los especímenes se realizó instrumentando con limas flexibles (K- Flexofile, Dentsply, Sweden) e irrigando con solución de hipoclorito de sodio al $2.5 \%$. La obturación radicular se practicó usando técnica de condensación lateral de gutapercha y cemento sellador con contenido de eugenol (cemento Grossman). El acceso coronario se selló con ionómero de vidrio convencional (ChemFil Superior, Dentsply, Tulsa, UK). Cada diente fue conservado en atmósfera de humedad de $95 \%$ a $37^{\circ} \mathrm{C}$ hasta su utilización.

Posteriormente, se removió la obturación coronaria y se talló una cavidad extendida desde la zona oclusal hasta la entrada de los conductos radiculares sin comprometer los rebordes marginales, simulando de esta forma condiciones propias de dientes endodonciados previas a la restauración.

El abordaje del conducto radicular se realizó con un ensanchador largo Peeso Reamer (Dentsply, Maillefer, Tulsa, UK) $N^{\circ} 2$, profundizando hasta aproximadamente $5 \mathrm{~mm}$ en el conducto y realizando un profuso lavado con agua presurizada.

Con disco de diamante se seccionaron las raíces a 5 milímetros del límite amelocementario, descartando la porción radicular. Con este mismo tipo de disco se inicia el corte en sentido bucolingual, para la división en dos mitades iguales (mesial y distal), efectuándose la división final por fractura de la pieza dentaria utilizando cincel y martillo, evitando así la contaminación de la zona de estudio.

\section{Tratamiento acondicionador de superficies dentinarias de grupos} experimentales.

Los especímenes de los cinco grupos experimentales recibieron distintos tratamientos acondicionadores utilizando para ello protocolos tradicionales de acondicionamiento y modificaciones de los mismos, según lo que se establece en la tabla 1.

Los puntos de medición se situaron en las zonas medias de las áreas a evaluar, dentina coronaria, dentina cameral y dentina del tercio cervical radicular, tal como se esquematiza en la figura1.

Cada muestra fue procesada para lograr punto crítico de sequedad, para luego ser montada y fijada en platina con pintura de plata y luego recubierta con oro 24 kilates en equipo Edwards, C150 Sputter, Coater, para ser examinada en microscopio electrónico de barrido (MEB), Jeol, modelo Jem 6380 LV., Japan. (Laboratorio de Microscopía Electrónica, Dirección de Investigación, Universidad de Concepción, Chile).

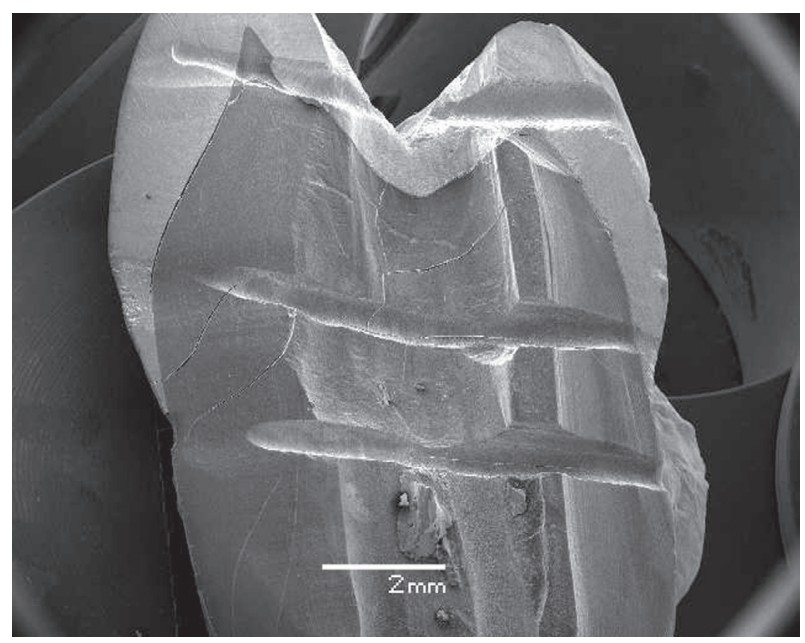

Figura 1. Fotografía electrónica de las tres zonas dentinarias a investigar

\section{LECTURA EN MEB}

Para el análisis en MEB de cada muestra se registraron 3 áreas de lectura en el centro de: Zona 1, dentina coronaria, desde el límite amelodentinario al inicio de la pared pulpar; Zona 2, cámara pulpar, entre techo y piso cameral, y Zona 3, tercio cervical radicular, desde el piso cameral hasta aproximadamente 1,5 milímetros del inicio del conducto vestibular. En cada sector se estudió:

Densidad Tubular: cantidad de túbulos visibles en la microfotografía de las muestras estudiadas en microscopio electrónico de barrido (MEB), en un área determinada.

Diámetro de los Túbulos: medida del espacio o luz obtenida en su sector más ancho.

Para el recuento en las lecturas en MEB, se fijó la magnificación en $1.000 x$ para densidad y $3.000 x$ para diámetro. Tanto la densidad como el diámetro se calcularon aplicando las fórmulas descritas por Cagidiaco y col. ${ }^{(10)}$ Schellemberg y col. ${ }^{(12)}$.

El número de túbulos dentinarios por $\mathrm{mm}^{2}$ se estableció según la fórmula: $X=n \times 106 / z$

Donde, $\mathrm{X}=$ número de túbulos dentinarios por $\mathrm{mm}^{2}, \mathrm{n}=$ número de túbulos en el área de prueba $z, y z$ = área de superficie del corte transversal en la fotomicrografía en $\mu \mathrm{m}^{2}$. Los distintos promedios fueron calculados para los diferentes sectores de cada diente. Las fotomicrografías se evaluaron en doble ciego. 
Diferentes Protocolos de Grabado Ácido en Dentina; Estudio Micromorfológico.

Tabla 1. Tratamiento acondicionador de la superficie dentinaria

\begin{tabular}{|c|c|c|c|}
\hline & $\begin{array}{c}\mathrm{H}_{3} \mathrm{PO}_{4} 35 \%\left(^{*}\right) \\
\text { Lavado profusamente con agua por } 5 \\
\text { seg. } y \text { secado por } 5 \text { seg. }\end{array}$ & $\begin{array}{c}\text { EDTA } 15 \%\left({ }^{* *}\right) \\
\text { Lavado con agua presurizada y secado } \\
\text { por } 5 \text { seg. }\end{array}$ & $\begin{array}{c}\text { NaOCI } 5 \%\left(^{(* *}\right) \\
\text { Lavado profusamente por } 5 \text { seg. y } \\
\text { secado por } 5 \text { seg .con agua }\end{array}$ \\
\hline Grupo Control & 5 segundos & & \\
\hline Grupo 1 & & 5 minutos, seguido de & Irrigación con $10 \mathrm{ml}$ con jeringa Luer \\
\hline Grupo 2 & 15 segundos & & \\
\hline Grupo 3 & 45 segundos & & \\
\hline Grupo 4 & 15 segundos, seguido de & & Irrigación por 60 seg. \\
\hline Grupo 5 & 45 segundos, seguido de & & Irrigación por 60 seg. \\
\hline * & $\mathrm{H}_{3} \mathrm{PO}_{4} 35 \%\left({ }^{*}\right)$ & \multicolumn{2}{|c|}{ 3M ESPE Dental Products, St. Paul, MN 55144-1000 USA } \\
\hline ** & EDTA Gel al $15 \%\left({ }^{* *}\right)$ & \multicolumn{2}{|l|}{ Alfadental, Chile } \\
\hline *** & $\mathrm{NaOCI} 5 \%\left(^{* * *}\right)$ & \multicolumn{2}{|l|}{ Alfadental, Chile } \\
\hline
\end{tabular}

\section{Análisis Estadísticos}

Los datos se describieron según sus valores promedios y su desviación estándar. Se incorporaron los valores máximos y mínimos observados. La comparación entre los tratamientos, se realizó aplicando un ANOVA a una vía de clasificación, utilizando la prueba de Bonferroni a posteriori, fijando un valor $p<0,05$ para significación estadística.

La comparación entre las secciones dentarias estudiadas, dentro de cada tratamiento, se analizó mediante prueba t de Student para datos apareados, fijando un valor $p<0,05$ para significación estadística.

Los datos fueron procesados con el programa Infostat versión profesional 2009.

Tabla 2. Densidad de túbulos dentinarios según acondicionamiento

\section{RESULTADOS}

\section{Densidad Tubular:}

Tanto a nivel de la zona coronaria, de cámara pulpar y de conducto radicular no se observaron diferencias significativas en la densidad de túbulos con los tratamientos aplicados. Tabla 2.

\section{Diámetro de los Túbulos:}

A nivel de las zonas 1 (coronaria) y 2 (cámara pulpar) no existen diferencias significativas entre el grupo control, grupos 1 y 2 ; pero sí entre éstos y los grupos 3,4 y 5 , ( $p<0,0001$ y $p=0,0005)$. Los mayores valores en el diámetro se obtuvieron cuando se acondicionó con ácido fosfórico al $35 \%$ + Hipoclorito de Sodio, como se puede observar en las figuras 2 y 3 , con $\times 1.000$ y x3.000, respectivamente.

\begin{tabular}{|c|c|c|c|c|c|}
\hline \multicolumn{6}{|c|}{ TÚBULOS-DENSIDAD $\left(\mathrm{n}^{\circ} / \mu \mathrm{m}^{2}\right)$ DIENTE JOVEN } \\
\hline Acondicionadores & Zona & Media & D.E. & Mín. & Máx. \\
\hline \multirow{3}{*}{ Control } & 1 & 30,00 & 7,89 & 22,00 & 45,00 \\
\hline & 2 & 43,44 & 13,48 & 25,00 & 70,00 \\
\hline & 3 & 29,44 & 16,67 & 11,00 & 63,00 \\
\hline \multirow{3}{*}{ EDTA $\times 5$ minutos $+\mathrm{NaOCl}$} & 1 & 25,89 & 6,97 & 20,00 & 43,00 \\
\hline & 2 & 49,22 & 14,37 & 26,00 & 73,00 \\
\hline & 3 & 37,44 & 14,30 & 16,00 & 59,00 \\
\hline \multirow{3}{*}{$\mathrm{H}_{3} \mathrm{PO}_{4} \times 15$ segundos } & 1 & 24,44 & 5,22 & 18,00 & 33,00 \\
\hline & 2 & 52,00 & 22,98 & 17,00 & 98,00 \\
\hline & 3 & 37,78 & 22,17 & 12,00 & 74,00 \\
\hline \multirow{3}{*}{$\mathrm{H}_{3} \mathrm{PO}_{4} \times 45$ segundos } & 1 & 28,80 & 8,35 & 22,00 & 43,00 \\
\hline & 2 & 41,20 & 10,26 & 27,00 & 52,00 \\
\hline & 3 & 23,40 & 5,55 & 19,00 & 32,00 \\
\hline \multirow{3}{*}{$\mathrm{H}_{3} \mathrm{PO}_{4} \times 15$ segundos $+\mathrm{NaOCl}$} & 1 & 30,00 & 7,14 & 19,00 & 43,00 \\
\hline & 2 & 53,33 & 18,51 & 35,00 & 84,00 \\
\hline & 3 & 34,00 & 9,50 & 20,00 & 56,00 \\
\hline \multirow{3}{*}{$\mathrm{H}_{3} \mathrm{PO}_{4} \times 45$ segundos $+\mathrm{NaOCl}$} & 1 & 29,80 & 5,54 & 21,00 & 35,00 \\
\hline & 2 & 38,80 & 10,52 & 28,00 & 54,00 \\
\hline & 3 & 22,00 & 7,78 & 15,00 & 34,00 \\
\hline
\end{tabular}

Prueba de ANOVA- Prueba a posteriori Bonferroni, fijando $p<0,05$ para significación estadística

1: $p=0,4163$

2: $p=0,4860$

3: $p=0,2522$ 


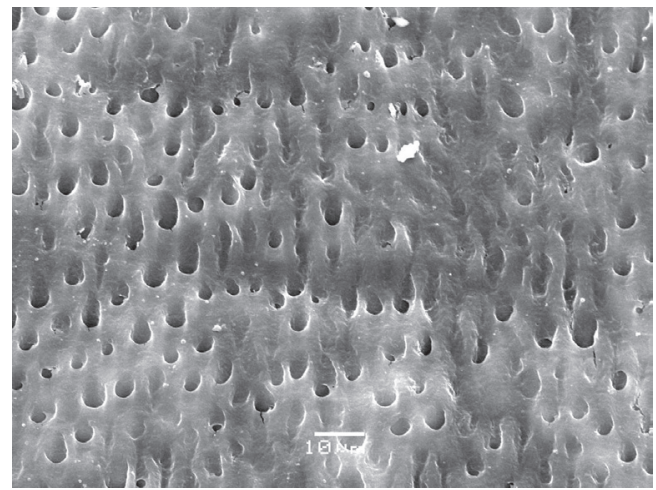

Figura 2A. Secuencia de microfotografías MEB (x1.000) ) dentina grabada con $\mathrm{H}_{3} \mathrm{PO}_{4}, 37 \%$ por 15 seg en dentina coronaria (zona 1 ).

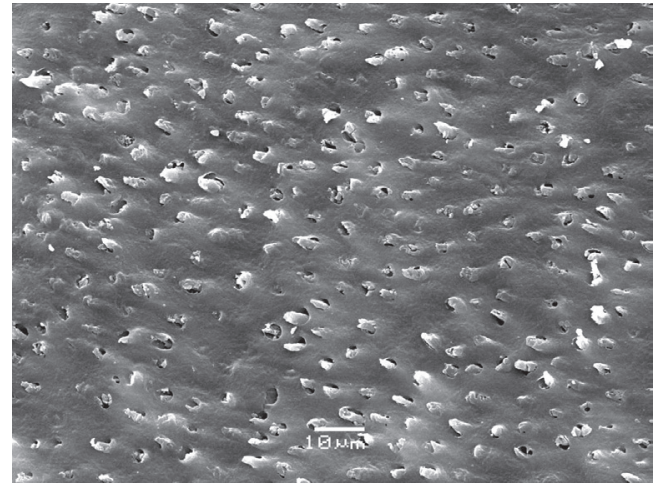

Figura 2B. Secuencia de microfotografías MEB (x1.000) dentina grabada con $\mathrm{H}_{3} \mathrm{PO}_{4}, 37 \%$ por 15 seg. en dentina pared cámara pulpar (zona 2).

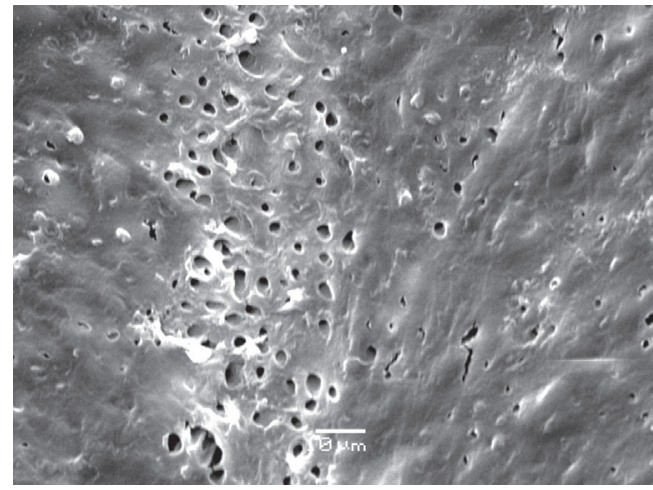

Figura 2C. Secuencia de microfotografías MEB (x1.000) dentina grabada con $\mathrm{H}_{3} \mathrm{PO}_{4}, 37 \%$ por 15 seg. en dentina tercio cervical del conducto radicular (zona 3 ).

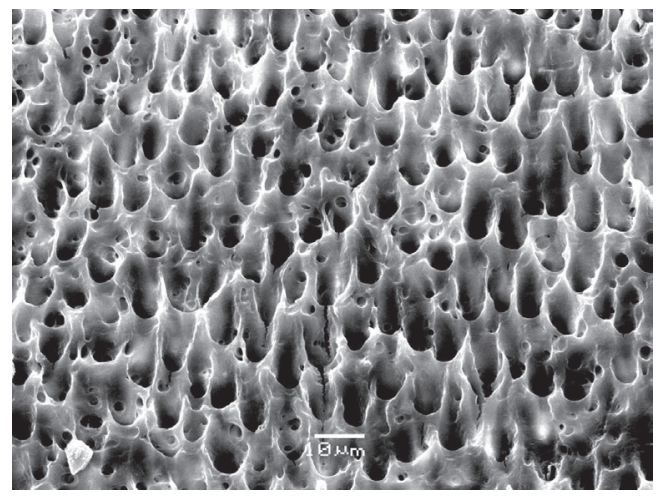

Figura 2D. Secuencia de microfotografías MEB (x1.000) dentina grabada con $\mathrm{H}_{3} \mathrm{PO}_{4}, 37 \%$ por $15 \mathrm{seg}+\mathrm{NaOCl}$, dentina coronaria (zona 1)

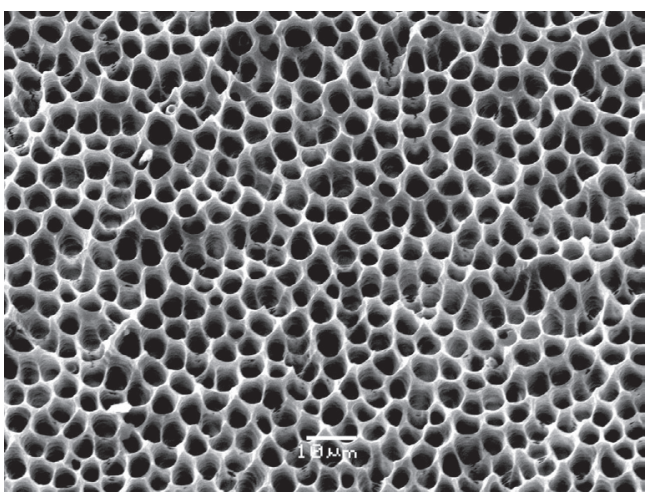

Figura 2E. Secuencia de microfotografías MEB (x1.000) dentina grabada con $\mathrm{H}_{3} \mathrm{PO}_{4}, 37 \%$ por $15 \mathrm{seg}+\mathrm{NaOCl}$, dentina pared cámara pulpar (zona 2 )

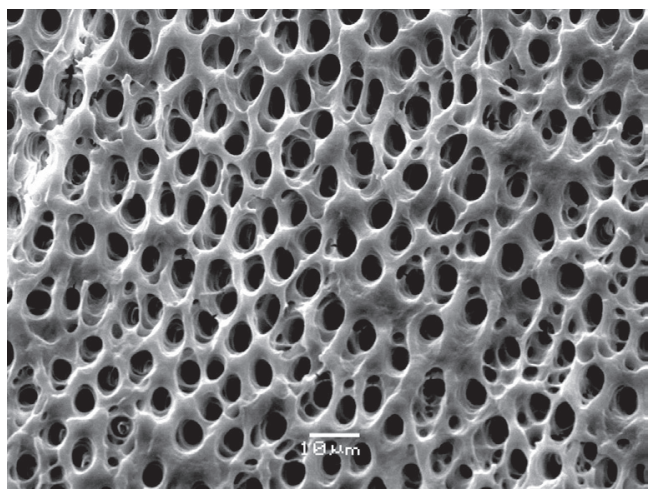

Figura 2F. Secuencia de microfotografías MEB (x1.000) dentina grabada con $\mathrm{H}_{3} \mathrm{PO}_{4}, 37 \%$ por $15 \mathrm{seg}+\mathrm{NaOCl}$, dentina tercio cervical del conducto radicular (zona 3 ).

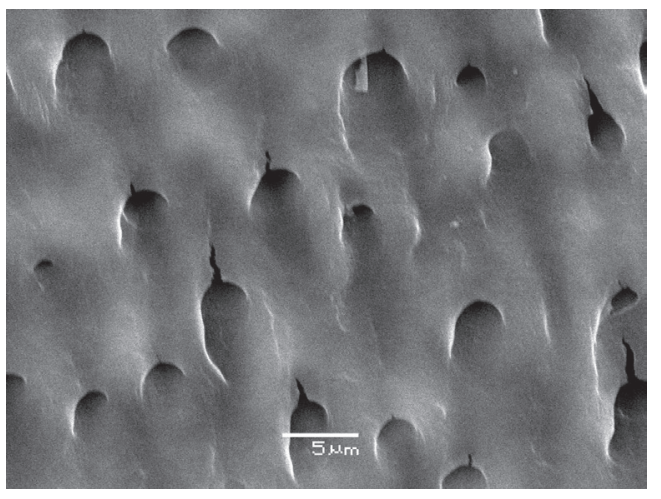

Figura 3A. Secuencia de microfotografías MEB (x3.000) dentina grabada con $\mathrm{H}_{3} \mathrm{PO}_{4}, 37 \%$ por 15 seg, dentina coronaria (zona 1).

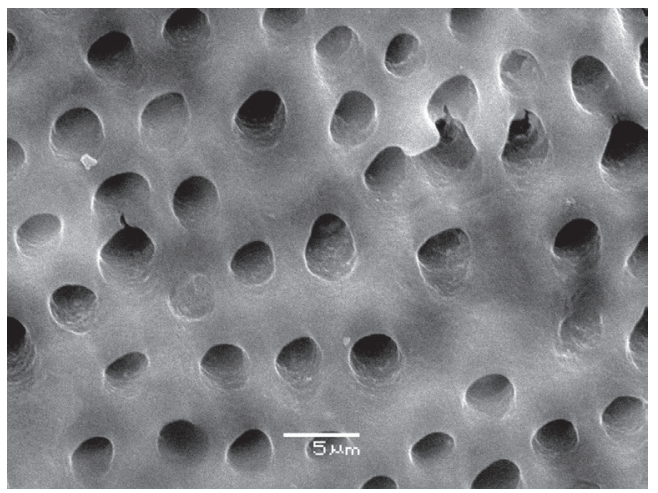

Figura 3B. Secuencia de microfotografías MEB (x3.000) dentina grabada con $\mathrm{H}_{3} \mathrm{PO}_{4}, 37 \%$ por $15 \mathrm{seg}$, dentina pared cámara pulpar (zona 2 ). 


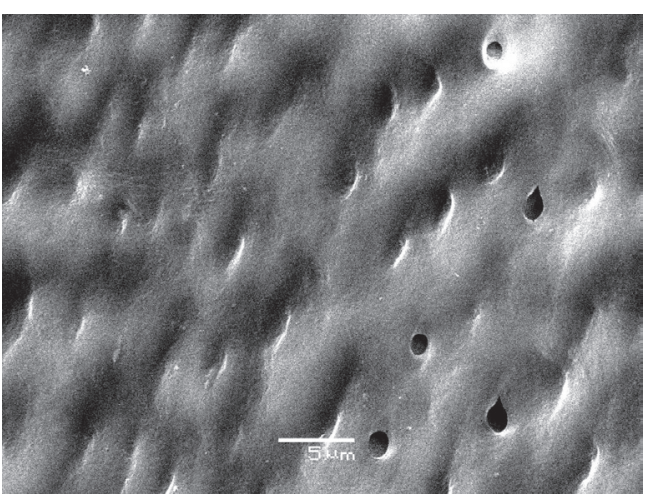

Figura 3C. Secuencia de microfotografías MEB (x3.000) dentina grabada con $\mathrm{H}_{3} \mathrm{PO}_{4}, 37 \%$ por $15 \mathrm{seg}$, dentina tercio cervical del conducto radicular(zona 3 ).

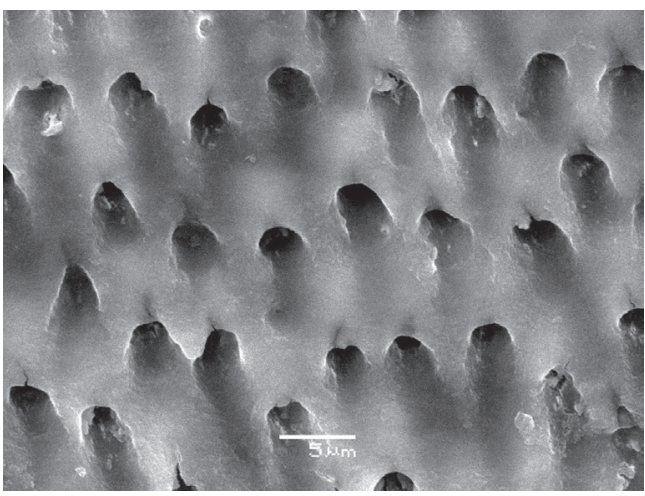

Figura 3D. Secuencia de microfotografías MEB (x3.000), dentina grabada con $\mathrm{H}_{3} \mathrm{PO}_{4}, 37 \%$ por $15 \mathrm{seg}+\mathrm{NaOCl}$, dentina coronaria (zona 1).

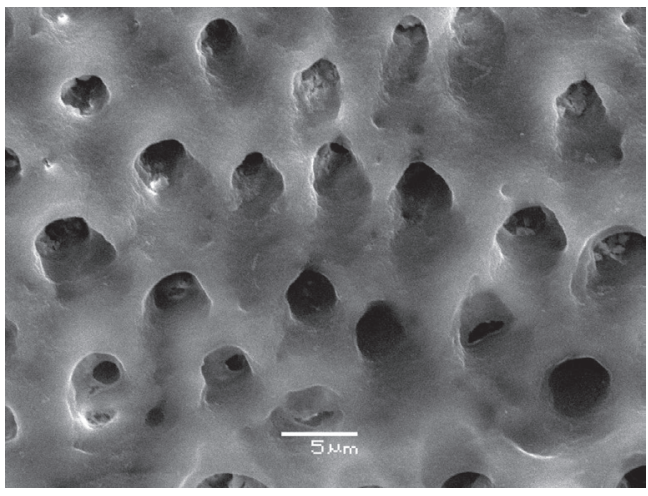

Figura 3E. Secuencia de microfotografías MEB (×3.000), dentina grabada con $\mathrm{H}_{3} \mathrm{PO}_{4}, 37 \%$ por $15 \mathrm{seg}+\mathrm{NaOCl}$, dentina pared cámara pulpar (zona 2).

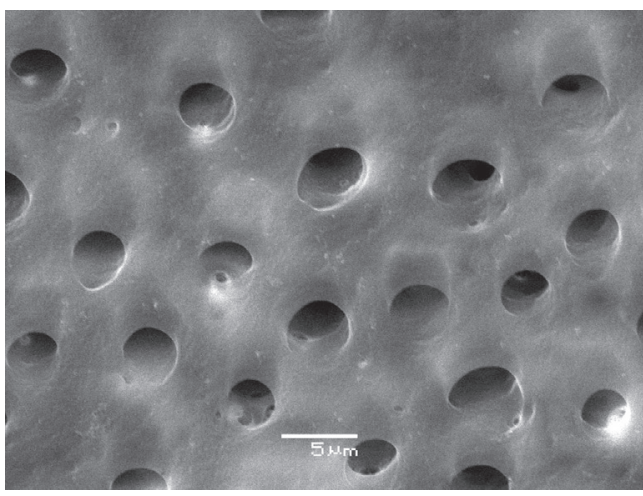

Figura 3F. Secuencia de microfotografías MEB (x3.000), dentina grabada con $\mathrm{H}_{3} \mathrm{PO}_{4}, 37 \%$ por $15 \mathrm{seg}+\mathrm{NaOCl}$, dentina tercio cervical del conducto radicular. (zona 3 ).
A nivel de zona inicial de conducto radicular, los diámetros tubulares mostraron diferencias significativas cuando se comparó el grupo control, grupos 2 y 4 ( $p=0,0040)$, en tanto, no se observaron diferencias significativas entre los grupos 1,3 y 5 , siendo este último el que mostró los mayores diámetros. Tabla 3.

Tabla 3. Diámetro de los túbulos dentinarios según acondicionamiento

\begin{tabular}{|c|c|c|c|c|c|}
\hline \multicolumn{6}{|c|}{ DIAMETRO-TÚBULOS $\left(\mu \mathrm{m}^{2}\right)$ DIENTE JOVEN } \\
\hline Acondicionadores & Zona & Med & D.E. & Mínim & Máximo \\
\hline \multirow{4}{*}{ Control } & 1 & 3,71 & 0,64 & 3,18 & 4,66 \\
\hline & 2 & 3,08 & 1,23 & 0,00 & 4,18 \\
\hline & 3 & 2,66 & 1,06 & 0,00 & 3,51 \\
\hline & 1 & 4,23 & 0,48 & 3,22 & 4,67 \\
\hline \multirow{3}{*}{$\begin{array}{c}\text { EDTA } \times 5 \\
\text { minutos }+\mathrm{NaOCl}\end{array}$} & 2 & 4,30 & 1,17 & 2,22 & 6,47 \\
\hline & 3 & 4,01 & 1,62 & 0,49 & 6,42 \\
\hline & 1 & 4,02 & 0,57 & 3,27 & 4,76 \\
\hline \multirow[t]{3}{*}{$\begin{array}{l}\mathrm{H}_{3} \mathrm{PO}_{4} \times 15 \\
\text { segundos }\end{array}$} & 2 & 3,74 & 0,37 & 2,96 & 4,18 \\
\hline & 3 & 2,83 & 1,51 & 0,00 & 4,80 \\
\hline & 1 & 5,20 & 0,96 & 4,22 & 6,54 \\
\hline \multirow{2}{*}{$\begin{array}{l}\mathrm{H}_{3} \mathrm{PO}_{4} \times 45 \\
\text { segundos }\end{array}$} & 2 & 4,78 & 1,15 & 2,78 & 5,44 \\
\hline & 3 & 3,31 & 0,76 & 2,00 & 3,89 \\
\hline \multirow{3}{*}{$\begin{array}{c}\mathrm{H}_{3} \mathrm{PO}_{4} \times 15 \\
\text { segundos }+ \\
\mathrm{NaOCl}\end{array}$} & 1 & 4,54 & 0,68 & 3,56 & 5,76 \\
\hline & 2 & 5,12 & 0,73 & 4,00 & 6,67 \\
\hline & 3 & 5,04 & 1,03 & 3,56 & 6,89 \\
\hline \multirow{3}{*}{$\begin{array}{c}\mathrm{H}_{3} \mathrm{PO}_{4} \times 45 \\
\text { segundos }+ \\
\mathrm{NaOCl}\end{array}$} & 1 & 6,90 & 1,85 & 5,33 & 9,22 \\
\hline & 2 & 5,11 & 0,96 & 3,67 & 6,33 \\
\hline & 3 & 4,42 & 1,71 & 3,00 & 7,33 \\
\hline
\end{tabular}

Prueba de ANOVA- Prueba a posteriori Bonferroni, fijando $p<0,05$ para significación estadística

1: $p<0,0001$ diferencias entre Control y $\mathrm{H}_{3} \mathrm{PO}_{4} \times 15$ segundos. $+\mathrm{NaOCl}$ y $\mathrm{H}_{3} \mathrm{PO}_{4} \times$ 45 segundos . $/ \mathrm{H}_{3} \mathrm{PO}_{4} \times 45$ segundos $+\mathrm{NaOCl}$

2: $p=0,0005$ diferencias entre Control, $\mathrm{H}_{3} \mathrm{PO}_{4} \times 15$ segundos. $+\mathrm{NaOCl}$ y $\mathrm{H}_{3} \mathrm{PO}_{4} \mathrm{X}$ 45 segundos . $/ \mathrm{H}_{3} \mathrm{PO}_{4} \times 45$ segundos $+\mathrm{NaOCl}$

3: $p=0,0040$ diferencias entre Control, $\mathrm{H}_{3} \mathrm{PO}_{4} \times 15$ segundos de $\mathrm{H}_{3} \mathrm{PO}_{4}$ $x 15$ seg. $+\mathrm{NaOCl}$

\section{DISCUSIÓN}

Las restauraciones utilizando sistemas adhesivos en dientes tratados endodónticamente ofrecen muchas ventajas si se comparan con materiales tradicionales no adhesivos ${ }^{(3)}$. Esto se debe a que una resina adherida al diente permite la trasmisión del estrés funcional a través de la interfase de unión, con un potencial refuerzo a la estructura debilitada del diente ${ }^{(4)}$.

Fusayama y col.(2) introdujeron el concepto de grabado total o técnica simultánea de grabado a esmalte y dentina con ácido fosfórico u otro agente ácido al tejido dentinario, como nuevo sustrato adhesivo. La heterogeneidad y el mayor contenido orgánico y acuoso de la dentina pueden ocasionar dificultades para conseguir adhesión con la técnica del grabado ácido, requiriéndose un mejor conocimiento de su microestructura y fisiología, así como de un constante desarrollo de múltiples sistemas adhesivos, lo que se produjo durante las últimas décadas ${ }^{(11,13)}$. Mjor y col.

(14) muestran una disminución en el número de túbulos dentinarios desde 57.600 túbulos $/ \mathrm{mm}^{2}$ en coronal a 19.600 túbulos $/ \mathrm{mm}^{2}$ en apical. Debido a esta heterogeneidad, la dentina se describe como un sustrato "dinámico" y, por lo tanto, representa un desafío para lograr adhesión ${ }^{(15)}$.

Los resultados obtenidos en este estudio han permitido establecer que el sustrato dentinario puede mostrar efectos, según las zonas evaluadas, de forma diferente cuando es sometido a la acción de distintos protocolos de acondicionamiento.

La densidad de los túbulos dentinarios ha sido estudiada ampliamente $^{(13,15,16)}$ y medida según profundidad dentinaria (límite 
amelodentinario a pared de la cámara pulpar) y también en dentina radicular coronal, media y apical. Las densidades registradas en este estudio coinciden con los resultados de los autores antes mencionados, considerando la profundidad dentinaria fijada para hacer las lecturas correspondientes. Si bien es cierto que no se logró establecer diferencias significativas entre ninguno de los grupos, sí fue evidente en las imágenes logradas en MEB, la existencia de túbulos dentinarios con diferencias importantes en el grado de apertura de los túbulos por remanentes de barro dentinario o restos de contaminantes producto del tratamiento endodóntico especialmente en las zonas dentinarias de camara pulpar y conducto readicular.

El diámetro de los túbulos varía desde 2.5 micrómetros cerca de la pulpa a 0.8 micrómetros en la unión amelodentinaria ${ }^{(15)}$. Para Gómez de Ferraris y Campos Muñoz ${ }^{(17)}$ alcanzan hasta 5 micrómetros cerca de la pulpa y un diámetro promedio de 1.7 micrómetros en la zona periférica.

Los valores de los diámetros obtenidos en este estudio no concuerdan con los que mencionan dichos autores ${ }^{(15,17)}$, sin embargo, esto era de esperar ya que para abordar los conductos radiculares hubo que tallar una cavidad de acceso cuyas paredes dentinarias fueron trasladadas a zonas diferentes a las utilizadas por ellos para hacer sus mediciones. Al revisar los valores de los diámetros de los túbulos dentinarios por zonas, se comprobó, tanto en los grupos controles como experimentales que los mayores diámetros correspondieron a la zona $1^{(18)}$, lo que se explica porque a este nivel los túbulos dentinarios cortados se mostraron en un alto porcentaje con una dirección oblicua, situación que por si sola expresa el aumento de su diámetro. Se sumó a esto el efecto de los diferentes protocolos de acondicionamiento.

Se pudo observar que en las zona 1 , dentina coronaria y zona 2 , cámara pulpar, los grupos 3, 4 y 5 fueron los que permitieron generar diferencias significativas respecto al subgrupo Control. Esto permitió inferir que el factor tiempo en la aplicación del ácido es la causa principal del aumento del diámetro de los túbulos, pero además, como el grupo 4 logró diferenciarse significativamente del Grupo Control, no así el grupo 2 , indicaría también la influencia del hipoclorito de sodio en el aumento del diámetro.

En la zona 3, dentina radicular, fue el grupo 4 el que logró valores con diferencias significativas respecto al grupo Control y al grupo 2 lo que nuevamente sugiere una participación activa del hipoclorito de sodio. Esto se debería a que la dentina puede ser acondicionada y activada por oxidación-desproteinización a través de la aplicación de Hipoclorito de Sodio al 5\%, logrando así aumentar el diámetro y tamaño de las ramas laterales y eliminar, de modo parcial, el colágeno de la dentina desmineralizada, generando así canales tridimensionales en el componente mineral dentinario, por donde difunden y se adhieren los monómeros hidrófilos-hidrófugos del sistema adhesivo de grabado total, formando una capa de dentina conocida como capa intermedia por desproteinización ${ }^{(19,20)}$.

De esta forma, queda de manifiesto que las zonas dentinarias sometidas al efecto ácido grabador mostraron cambios de manera diferente, lo que concuerda con Marshall(21), quien concluyó que la dentina presenta propiedades y una naturaleza con variaciones regionales importantes, pero además, que las interacciones entre la dentina y el acondicionador dependen del agente que se aplique, de su concentración, $\mathrm{pH}$ y del tiempo de aplicación.

Se evidencia también en este estudio que las áreas dentinarias involucradas directamente durante el tratamiento endodóntico, en especial en contacto con el cemento sellador del conducto (Cemento de Grossman), mostraron con cierta frecuencia restos del cemento dentro de los túbulos destinarios, aun después de haber realizado los protocolos de grabado con tiempos normales de 15 segundos, pero que esta tendencia se redujo cuando se aumentó el tiempo de grabado y/o se agregó el efecto del hipoclorito de sodio ${ }^{(22)}$. Tales consideraciones permiten concordar con Bottino y col.(23), quienes refieren que podría producirse una reducción de la fuerza de adhesión de la resina y disminución de la adaptación marginal, por lo que la contaminación previa del tejido dentinario podría ser visto como factor adicional que influiría en el proceso de adhesión ${ }^{(24)}$. Samah Saker y col. ${ }^{(25)}$, igualmente confirman que lograr superficies limpias de dentina del conducto radicular después de la preparación mecánica es un paso crucial para la retención óptima de un poste, particularmente cuando se usa cemento de resina.

Queda en evidencia en este estudio que el aumento de la agresividad de la técnica de grabado ya sea aumentando el tiempo de grabado (protocolo 3) o agregando el efecto del hipoclorito de sodio (protocolo 4) o utilizando ambos recursos (protocolo 5) logran cambios importantes en las tres zonas estudiadas respecto al grabado tradicional de grabado con ácido fosfórico por 15 segundos, siendo el protocolo 4 el que logra efectos más regulares.

Nakabayashi y col.(26), han establecido que la infiltración de los monómeros de resina en el colágeno expuesto después del grabado ácido es incompleta conformando espacios dentro de la capa híbrida y permitiendo también nanofiltración, lo que puede conducir al deterioro de la adhesión entre monómeros de resina y dentina. Otros estudios han demostrado que la capa de colágeno no contribuye significativamente a la resistencia de la unión interfacial de la resina a la dentina y que la adhesión con algunas resinas puede aumentar si la capa de colágeno previamente desmineralizada se disuelve primero y se elimina por un agente proteolítico no específico como es el caso del $\mathrm{NaOCl}\left({ }^{(27,28,29)}\right.$. Mao $\mathrm{H}$. y col. ${ }^{(30)}$, concluyeron en su estudio que los tratamientos de dentina radicular con $\mathrm{NaOCl}$ después del grabado de la dentina dieron como resultado aumentos significativos en las resistencias de unión por empuje comparados con los controles, independientemente del sitio del conducto radicular analizado y del cemento resina usado, lo que concuerda con la propuesta y resultados de los protocolos de grabado de nuestro estudio.

Carvalho y col.(31), informaron en su estudio un bajo rendimiento en fuerzas de retención de postes cementados cuando los dientes fueron acondicionados con ácido ortofosfótico al $37 \%$ por 15 segundos, ya que este ácido, comparado con otros protocolos, no eliminó los contaminantes que se alojan en diferentes localizaciones del conducto radicular, lo que comprometería la difusión de los monómeros del sistema adhesivo, afectando la resina de cementación.

Según Vichi ${ }^{(32)}$, la adhesión a dentina radicular es esencialmente micromecánica, basado en la infiltración de la dentina desmineralizada y la formación de tags de resina y ramas laterales adhesivas. Un protocolo de acondicionamiento que lleve a una mejora en el patrón de superficie podría conducir a la formación de mayor densidad de tags de resina y, por lo tanto, una mayor superficie de adhesión, lo cual afirma la importancia de patrones micromorfológicos que sugieran una buena formación de tags de resina y ramas laterales en zonas dentinarias complejas, como cámara pulpar y tercio cervical radicular. Así también, es en estas zonas donde aún pareciera tener un mejor comportamiento la adhesión con sistemas adhesivos de grabado total, tal como lo plantea Calixto y col(33). La menor cantidad de túbulos dentinarios y la estructura irregular de la dentina secundaria en las paredes del canal radicular resultan en una menor penetración de los adhesivos en la porción de la dentina radicular en comparación con la dentina coronaria(34), lo cual también se puede deducir de los resultados de este estudio.

\section{CONCLUSIONES}

Los resultados de este estudio aportan datos que ponen de manifiesto el comportamiento clínico heterogéneo de las distintas zonas dentinarias, luego de la aplicación de diferentes protocolos de acondicionamiento para la adhesión a dentina con técnica de grabado total.

Específicamente, los resultados permiten plantear que un protocolo tradicional de acondicionamiento con ácido ortofosfórico al 35\% por 15 segundos no asegura un grabado uniforme de las tres zonas dentinarias estudiadas. Sin embargo, cuando se aumenta el tiempo de grabado y/o se incorpora hipoclorito de sodio al $5 \%$ por 1 minuto como agente desproteinizante, los efectos del grabado sobre densidad y diámetro de los túbulos dentinarios son más homogéneos y predecibles.

\section{CONFLICTO DE INTERÉS}

Los autores no tenemos conflictos de interés. 


\section{Bibliografía}

1. Nakabayashi N. Pashley DH. Hybridization of dental hard tissues. $1^{\text {a }}$ Ed. Tokio, Japan: Quintessence Publishing 1998: pp: 82-83.

2. Fusayama T, Nakamura M, Kurosaki N, Iwaku M. Non-Pressure adhesion of a new adhesive restorative resin. J Dent Res. 1979;58(4):1364-70.

3. Ozturk $\mathrm{B}$, Ozer F. Effect of $\mathrm{NaOCl}$ on bond strengths of bonding agents to pulp chamber lateral walls. J Endod. 2004;30(5);362-5.

4. Belli S, Zhang Y, Pereira PNR, Ozer F, Pashley DH. Regional bond strengths of adhesive resins to pulp chamber dentin. J Endod. 2001;27(8):527-32.

5. Aasen SM. History of dentinal bonding. Esthet Dent Update 1990;1:43-46.

6. Priotto EG, Uribe Echevarría DI, Uribe Echevarría J. Morphological and numerica characteristics of dentine tubules destined to adhesion. J Dental Res. Divisional Abstracts. 1995;74:734-38.

7. Causton BE. Improved bonding of composite restorative to dentin. A study in vitro of the use of commercial halogenated phosphate ester. Br Dent J. 1984;156(3):93-5 8. Giannini M, Carvalho RM, Martins LR, Dias CT, Pashley DH. Influence of tubule density and area of solid dentin on bond strength of two adhesive systems to dentin. J Adhes Dent. 2001:3(4):315-24.

9. Tanumiharja M,. Burrow ME, Tyas MJ. Microtensile bond strengths of seven dentin adhesive systems. Dent Mater. 2000;16(3):180-7.

10. Cagidiaco MC, Ferrari M, Vichi A, Davidson CL. Mapping of tubule and intertubule surface area available for bonding in class $\mathrm{V}$ and class II preparation. J Dent. 1997;25(5):379-89.

11. Marshall GW, Marshall S, Kinney JH, Balooch M. The dentin substrate: structure and properties related to bonding. J Dent. 1997;25 (6):441-58.

12. Schellenberg $U$, Krey G, Bosshardt D, et al. Density of dentinal tubules at the pulpal of human permanent premolars and third molars. J Endod. 1992;18:104-109. 13. Korkmaz, Gurgan S, Firat E, Nathanson D. Effect of adhesives and thermocycling on the shear bond strength of a nano-composite to coronal and root dentin. Oper Dent. 2010;35(5):522-529.

14. Mjor IA, Nordahl I. The density and branching of dentinal tubules in human teeth. Arch Oral Biol. 1996;41(5):401-12.

15. Esclassan E, Grégorie G, Cournot M. Morphological study of fiber-reinforced post-bonding system-root dentin interface by evaluation of two bonding systems. J Dent. 2008;36(3):204-213.

16. Ferrari M, Mannocci F, Vichi A, Cagidiaco MC, Mjor IA. Bonding to root canal: structural characteristic of the substrate. Am J Dent. 2000;13(5): 255-60.

17. Gómez de Ferraris, M., Campos Muñoz, A. Histología y embriología bucodental. Madrid: Editorial Médica Panamericana; 2002. p.238-40.

18. Schiltz-Taing M, Wang Y, Suh B, Brown D, Chen L. Effect of tubular orientation on the dentin bond strength of acidic self-etch adhesive. Oper Dent. 2011;36(1):86-91.
19. Uceda-Gomez N, Reis A, Rocha M, Dourado A, Rodrigues E. Effect of sodium hypochlorite on the bond strength of an adhesive system to superficial and deep dentin. J Appl Oral Sci. 2003;11(3):223-8.

20. Perdigao J, Lopes M, Geraldeli S, Lopes G, García-Godoy F. Effect of a sodium hypochlorite gel on dentin bonding. Dent Mater. 2000;16: 311-323.

21. Marshall Jr. Dentin: Microstructure and characterization. Quintessence Int 1993;24(9):606-17.

22. Jordão-Basso KC, Kuga MC, Bandéca MC, Duarte MA, Guiotti FA. Effect of the time-point of acid etching on the persistence of sealer residues after using different dental cleaning protocols. Braz Oral Res. 2016;30(1):e133.

23. Bottino MA, Quintas AF, Miyashita E, Giannini V. Estética en rehabilitación oral. Metal free. Brasil. Editorial Artes Médicas Latinoamérica, 2001.

24. Kinney JH, Baloch M, Haupt Jr DL, Marshall SJ, Marshall Jr GW. Mineral distribution and dimensional changes in human dentin during demineralization. $J$ Dent Res. 1995;74(5):1179-84.

25. Samah S, Ózcan M. Retentive stregth of fiber-reinforced composite posts with composite resin cores: Effect of remaning coronal structure and root canal dentin conditioning protocolos. J Prosthet Dent. 2005;114:856-61.

26. Nakabayashi N. Kolima K, MAsuhara E. The promotion of adhesion by the infiltration of monomers into tooth substrates. J Biomed Mater Res. 1982;16:265-73. 27. De Munck J, Vargas M, Van Landuyt K, Hikita K, Lambrechts P, VanMeerbeek $B$. Bonding on an auto-adhesive luting material to enamel and dentin. Dent Mater. 2004;20:963-71.

28. Eldeniz AU, Erdemir A, Belli S. Shear bond strength of three resin based scalers to dentin with and without the smear layer. J Endod 2005;31:293-6.

29. Monticelli F, Osorio R, Mazzitelli C, Ferrari M, Toledado M. Limited decalcificacion/ diffusion of self-adhesive cements into dentin. J Dent Res. 2008;87:974-9.

30. Mao H, Chen Y, Yip KH, Smales RJ. Effect of three radicular dentine treatments and two luting cements on the regional bond strength of quartz fibre posts. Clin Oral Investig. 2011;15(6):869-78.

31. Carvalho RM, Tjäderhane L, Manso AP, Carrilho MR, Carvalho CAR. Dentin as a bonding subsrtrate. Endod Topics 2012;21:62-88.

32. Vichi A, Grandini S, Davidson C, Ferrari M. An SEM evaluation of several adhesive systems used for bonding fiber posts under clinical conditions. Dent Mater 2002;18(7):495-502.

33. Calixto LR, Bandéca MC, Clavijo V, Andrade MF, Vaz LG, Campos EA. Effect of resin cement system and root region on. the push-out bond strength of a translucent fiber post. Oper Dent. 2012;37(1), 80-6.

34. Schiltz-Taing M, Wang Y, Suh B, Brown D, Chen L. Effect of tubular orientation on the dentin bond strength of acidic self-etch adhesive. Oper Dent. 2011;36(1):86-91. 